\title{
Simulation of elastic scattering with a coupled FMBE-FE approach
}

\author{
D. Brunner, M. Junge \& L. Gaul \\ Institute of Applied and Experimental Mechanics, University of Stuttgart, \\ Germany
}

\begin{abstract}
In this paper scattering problems with elastic obstacles that are hit by an incident acoustic wave are discussed. Underwater acoustics mainly differs from air acoustics in the fact that a strong coupling scheme between the structural part and the acoustic domain is necessary. Such a scheme is discussed, using the fast multilevel multipole boundary element method (FMBEM) to model the exterior acoustic fluid and the finite element method (FEM) to model the structural part. To obtain a high flexibility, an interface to a commercial FE package is established. For a high efficiency, an iterative solver with preconditioning is applied. The numerical results are compared with an analytical solution for a model problem.

Keywords: elastic scattering, fluid-structure interaction, FE-BE coupling, fast boundary element method, multipole method.
\end{abstract}

\section{Introduction}

Fluid-structure interaction deals with the mutual influence of an acoustic and a structural domain. Since water is assumed as acoustic fluid with a high density a the structure is thin-walled, the feedback of the acoustic pressure onto the structure has to be taken into account. As a consequence, fully coupled simulation schemes have to be applied, which are computationally more expensive since a structural problem and an acoustic problem have to be solved simultaneously. In this paper, scattering problems are discussed where the structure is excited by an incident wave. The structural part is favorably modeled using the FEM. For the infinite fluid domain, the BEM is applied. To overcome the drawback of fully populated $\mathrm{BE}$ matrices, the fast multipole method is used. 
The paper starts with the governing equations of the multifield problem. Then, a FE discretization of the structural problem is presented. After this, the BEM is introduced to model the exterior acoustic domain. Special attention is paid to the fast multipole implementation. The remaining sections discuss the iterative solution of the coupled problem and the investigation of a model problem.

\section{Governing equations of the multifield problem}

The governing equations of the fluid-structure interaction problem are presented in the frequency domain with the time harmonic behavior $\mathrm{e}^{-\mathrm{i} \omega t}$, where $\omega=2 \pi f$ denotes the angular frequency. The structural domain $\Omega_{\mathrm{s}}$ (cf. Fig. 1) is assumed to be linear elastic with the Lamé constants $\lambda$ and $\mu$. The material is homogeneous with the structural density $\varrho_{\mathrm{s}}$. The corresponding elastodynamic problem for the displacements $u$ is given by

$$
\begin{aligned}
\omega^{2} \varrho_{\mathrm{s}} u(x)+\mu \Delta u(x)+(\lambda+\mu) \operatorname{grad} \operatorname{div} u(x) & =0 & & \text { for } x \in \Omega_{\mathrm{s}}, \\
T u(x) & =t_{\mathrm{s}}=0 & & \text { for } x \in \Gamma_{\mathrm{s}},
\end{aligned}
$$

and additionally a transmission condition which will be introduced later by eqn. (5). The Laplacian is denoted by $\Delta$ and $T$ represents the traction operator. The time harmonic Helmholtz equation is applied to describe the acoustic pressure $p$ in the fluid domain $\Omega_{\mathrm{a}}$

$$
\begin{aligned}
& \Delta p(x)+\kappa^{2} p(x)=0 \quad \text { for } x \in \Omega_{\mathrm{a}}, \\
& \quad\left|\frac{\partial p}{\partial R}-\mathrm{i} \kappa p\right|<\frac{c_{\mathrm{f}}}{R^{2}} \quad \text { for } R=|x| \rightarrow \infty,
\end{aligned}
$$

where an additional transmission condition is given by eqn. (6). The circular wave number is denoted by $\kappa=\frac{\omega}{c_{\mathrm{f}}}$ and the speed of sound is $c_{\mathrm{f}}$. Equation (4) is called Sommerfeld radiation condition, which ensures an outgoing wave within the exterior acoustic domain [1]. A strong coupling scheme is represented by the two transmission conditions

$$
\begin{aligned}
& T u(x)=t_{\mathrm{f}}(x)=-p(x) n_{x} \quad \text { for } x \in \Gamma_{\mathrm{I}}, \\
& q(x):=\frac{\partial p(x)}{\partial n_{x}}=\omega^{2} \varrho_{\mathrm{f}} u(x) n_{x} \quad \text { for } x \in \Gamma_{\mathrm{I}},
\end{aligned}
$$

where the acoustic flux $q$ is introduced. In case of a scattering problem, the overall acoustic field in $\Omega_{\mathrm{a}}$ (cf. Fig. 1) is the superposition of the incident and the scattered field [2]

$$
p=p^{\text {inc }}+p^{\mathrm{s}} \text { and } q=q^{\text {inc }}+q^{\mathrm{s}} .
$$

In what follows, the incident field ( $)^{\text {inc }}$ is prescribed. It is caused by a source, e.g. a plane wave, and corresponds to the sound field in the absence of the structure. The scattered field is denoted by ( ) ${ }^{\mathrm{s}}$. 


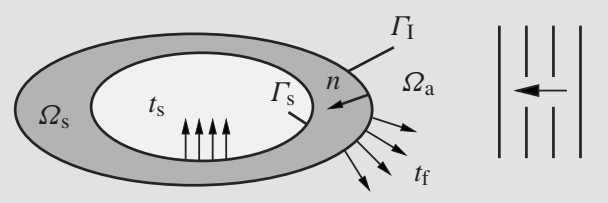

Figure 1: Elastic scattering: The exterior acoustic domain $\Omega_{\mathrm{a}}$ is in contact with the structure domain $\Omega_{\mathrm{s}}$ on the fluid-structure interface $\Gamma_{\mathrm{I}}$. The surface stress vector of the fluid is denoted by $t_{\mathrm{f}}$. The structural stress vector $t_{\mathrm{s}}$ is assumed to be zero.
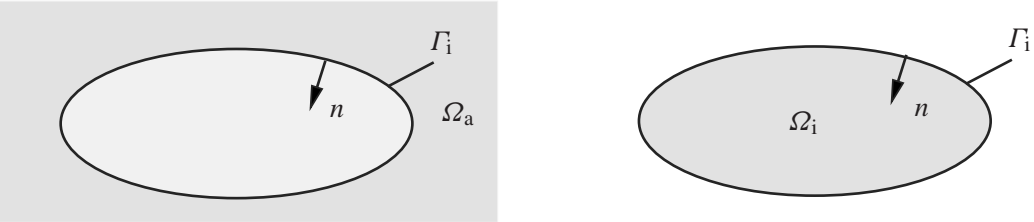

Figure 2: Exterior acoustic domain $\Omega_{\mathrm{a}}$ (left) and associated interior domain $\Omega_{\mathrm{i}}$ (right). In both cases, the normal $n$ is assumed to point inwards.

\section{FE formulation for the structural problem}

The FE discretization of the structural problem eqns. (1), (2) and (5) results in a system of linear equations

$$
\underbrace{\left(-\omega^{2} \boldsymbol{M}_{\mathrm{s}}-\mathrm{i} \omega \boldsymbol{D}_{\mathrm{s}}+\boldsymbol{K}_{\mathrm{s}}\right)}_{=: \boldsymbol{K}_{\mathrm{FE}}} \boldsymbol{u}=\boldsymbol{f}_{\mathrm{f}},
$$

where $\boldsymbol{M}_{\mathrm{s}}$ and $\boldsymbol{K}_{\mathrm{s}}$ denote the mass matrix and the stiffness matrix, respectively. Damping may be incorporated by the damping matrix $\boldsymbol{D}_{\mathrm{s}}$. However, structural damping is neglected in this paper. The nodal force vector $\boldsymbol{f}_{\mathrm{f}}$ represents the forces due to the fluid pressure and is discussed later in Section 5. The finite element package ANSYS is utilized to set up the matrices $\boldsymbol{M}_{\mathrm{S}}, \boldsymbol{K}_{\mathrm{s}}$. They are imported into the research code by a binary interface [3]. This data exchange has to be done only once for a given model, as $\boldsymbol{M}_{\mathrm{s}}$ and $\boldsymbol{K}_{\mathrm{s}}$ are frequency independent. Typically, shell elements with rotational degrees of freedom are applied for thin structures. Thus, each node has six degrees of freedom, which are $\left\{u_{x}, u_{y}, u_{y}, \theta_{x}, \theta_{y}, \theta_{z}\right\}$.

\section{BE formulation for the acoustic problem}

First, the exterior acoustic problem as depicted in Fig. 2 (left) is investigated. After this, a generalization for scattering problems is presented. 
Applying Green's second theorem to a weighted residual form of eqn. (3) and using the fundamental solution

$$
P(x, y)=\frac{\mathrm{e}^{\mathrm{i} \kappa r}}{4 \pi r}
$$

with $r=|x-y|$ yields the representation formula

$$
p(x)=\int_{\Gamma_{\mathrm{i}}} P(x, y) \frac{\partial p(y)}{\partial n_{y}} \mathrm{~d} s_{y}-\int_{\Gamma_{\mathrm{i}}} \frac{\partial P(x, y)}{\partial n_{y}} p(y) \mathrm{d} s_{y}, \quad x \in \Omega_{\mathrm{a}}
$$

which is valid for $x$ within the acoustic domain. The boundary integral equation is obtained by shifting $x$ onto the smooth boundary. With the definition of the acoustic flux $q(x):=\frac{\partial p(x)}{\partial n_{x}}$ one obtains

$$
\frac{1}{2} p(x)=\underbrace{\int_{\Gamma_{\mathrm{i}}} P(x, y) q(y) \mathrm{d} s_{y}}_{=:(V q)(x)}-\underbrace{\int_{\Gamma_{\mathrm{i}}} \frac{\partial P(x, y)}{\partial n_{y}} p(y) \mathrm{d} s_{y}}_{=:(K p)(x)}, \quad x \in \Gamma_{\mathrm{i}} .
$$

The single layer potential is denoted by $V$ and the double layer potential by $K$, respectively. Analogously, the hypersingular boundary integral equation is derived by an additional derivative with respect to the normal $n_{x}$

$$
\frac{1}{2} q(x)=\underbrace{\int_{\Gamma_{\mathrm{i}}} \frac{\partial P(x, y)}{\partial n_{x}} q(y) \mathrm{d} s_{y}}_{=:\left(K^{\prime} q\right)(x)}-\underbrace{\frac{\partial}{\partial n_{x}} \int_{\Gamma_{\mathrm{i}}} \frac{\partial P(x, y)}{\partial n_{y}} p(y) \mathrm{d} s_{y}}_{=:-(D p)(x)}, \quad x \in \Gamma_{\mathrm{i}}
$$

where $K^{\prime}$ denotes the adjoint double layer potential and $D$ is the hypersingular operator.

For exterior acoustic problems, neither the singular boundary integral equation (11) nor the hypersingular boundary integral equation (12) have a unique solution for all frequencies. One possibility to overcome the problem is the approach of Burton and Miller [4], which uses a linear combination of both integral equations (11) and (12)

$$
\left(\frac{1}{2} I+K-\alpha D\right) p(x)=\left(V-\frac{1}{2} \alpha I+\alpha K^{\prime}\right) q(x)
$$

where typically $\alpha=-\mathrm{i} / \kappa$ is chosen for a good condition number.

To derive a boundary integral representation for the scattering problem, eqn. (13) is applied to the scattered pressure $p^{\mathrm{s}}$ in $\Omega_{\mathrm{a}}$ (see Fig. 2 left)

$$
\frac{1}{2} p^{\mathrm{s}}(x)+\left(K p^{\mathrm{s}}\right)(x)-\alpha\left(D p^{\mathrm{s}}\right)(x)=\left(V q^{\mathrm{s}}\right)(x)-\frac{1}{2} \alpha q^{\mathrm{s}}(x)+\alpha\left(K^{\prime} q^{\mathrm{s}}\right)(x) .
$$


The same is done for $p^{\text {inc }}$ in the interior domain $\Omega_{\mathrm{i}}$ (see Fig. 1 right) in absence of the structure

$$
\begin{aligned}
- & \frac{1}{2} p^{\mathrm{inc}}(x)+\left(K p^{\mathrm{inc}}\right)(x)-\alpha\left(D p^{\mathrm{inc}}\right)(x) \\
& =\left(V q^{\mathrm{inc}}\right)(x)+\frac{1}{2} \alpha q^{\mathrm{inc}}(x)+\alpha\left(K^{\prime} q^{\mathrm{inc}}\right)(x) .
\end{aligned}
$$

Please note, the integral-free terms need to be multiplied by minus one, since the normal points into the fluid. Adding eqn. (14) to eqn. (15) and using eqn. (7) yields

$$
\begin{aligned}
& \frac{1}{2} p(x)+(K p)(x)-\alpha(D p)(x)-\left((V q)(x)-\frac{1}{2} \alpha q(x)+\alpha\left(K^{\prime} q\right)(x)\right) \\
& \quad=p^{\text {inc }}(x)-\alpha q^{\text {inc }}(x) .
\end{aligned}
$$

The Galerkin method is used to obtain an algebraic system of equations. Hence, eqn. (16) is tested with linear test functions $v$ on the interface $\Gamma_{\mathrm{I}}$. The pressures $p$ and $p^{\text {inc }}$ are interpolated with piecewise linear shape functions whereas constant shape functions are used for the fluxes $q$ and $q^{\text {inc }}$. The resulting algebraic system of equations reads

$$
\underbrace{\left(\frac{1}{2} \boldsymbol{M}+\boldsymbol{K}-\alpha \boldsymbol{D}\right)}_{\boldsymbol{K}_{\mathrm{BE}}} \boldsymbol{p}-\underbrace{\left(\boldsymbol{V}-\frac{1}{2} \alpha \boldsymbol{M}^{\prime}+\alpha \boldsymbol{K}^{\prime}\right)}_{\boldsymbol{C}_{\mathrm{BE}}^{q}} \boldsymbol{q}=\underbrace{\boldsymbol{M} \boldsymbol{p}^{\mathrm{i} n c}-\alpha \boldsymbol{M}^{\prime} \boldsymbol{q}^{\mathrm{i} n c}}_{\boldsymbol{b}_{\mathrm{BE}}},
$$

where $\boldsymbol{p}^{\text {inc }}$ is the vector with the nodal incident pressures and $\boldsymbol{q}^{\text {inc }}$ is the vector with the incident flux on the elements due to the scattering source.

\subsection{Implementation with the fast multipole method}

Matrices $\boldsymbol{K}_{\mathrm{BE}}$ and $\boldsymbol{C}_{\mathrm{BE}}^{q}$ in eqn. (17) are fully populated for standard boundary element methods, which results in an expense of quadratic order. To overcome this drawback, the fast multipole method is applied. For the introduced operators, one typically has to evaluate potentials of the type

$$
\Phi\left(x_{b}\right)=\sum_{a=1}^{A} \frac{\mathrm{e}^{\mathrm{i} \kappa\left|x_{b}-y_{a}\right|}}{\left|x_{b}-y_{a}\right|} q_{a},
$$

where $q_{a}$ denotes the source strengths of $A$ sources. The multipole algorithm sets up a clustering and sums up the contribution of all sources $q_{a}$ in the center $z_{a}$ of a cluster (see Fig. 3). At the next step, this so-called far-field signature is translated to the center $z_{b}$ of the other clusters and from there finally distributed to $x_{b}$. 


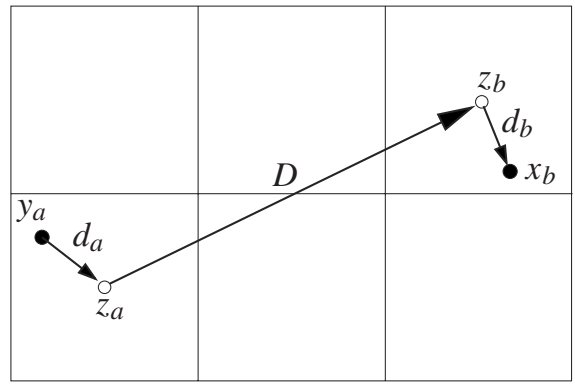

Figure 3: Clustering and splitting up of the vector between load point and field point into three parts.

From a mathematical point of view, the separation of the distance $\left|x_{b}-y_{a}\right|$ in the fundamental solution succeeds by using the diagonal form of the multipole expansion [5]

$$
\frac{\mathrm{e}^{\mathrm{i} \kappa\left|x_{b}-y_{a}\right|}}{\left|x_{b}-y_{a}\right|}=\frac{\mathrm{i} \kappa}{4 \pi} \sum_{l=0}^{\infty}(2 l+1) \mathrm{i}^{l} h_{l}^{(1)}(\kappa|D|) \int_{\mathbb{S}^{2}} \mathrm{e}^{\mathrm{i} \kappa\left(d_{a}+d_{b}\right) \cdot s} P_{l}(s \cdot \hat{D}) \mathrm{d} s,
$$

with the Hankel functions $h_{l}$ and the Legendre polynomials $P_{l}$. The vectors which are local to the clusters are denoted by $d_{a}$ and $d_{b}$ (see fig. 3 ), whereas $D$ is defined by the centers of two interacting clusters. The unit distance vector is defined by $\hat{D}=D /|D|$. The integral over the unit sphere $\mathbb{S}^{2}$ is approximated by Gauss point quadrature using discrete values of the far-field directions $s$ [5]. Since one can not compute an infinite sum, the series has to be truncated. In this case the integration over the unit sphere $\mathbb{S}^{2}$ and the summation can be interchanged. Introducing the translation operator

$$
M_{L}(s, D)=\sum_{\ell=0}^{L}(2 \ell+1) \mathrm{i}^{\ell} h_{\ell}^{(1)}(\kappa|D|) P_{\ell}(s \cdot \hat{D}),
$$

the original potential eqn. (18) can now be expressed in the form

$$
\Phi\left(x_{b}\right) \approx \frac{\mathrm{i} \kappa}{4 \pi} \int_{\mathbb{S}^{2}} \mathrm{e}^{\mathrm{i} \kappa d_{b} \cdot s} M_{L}(s, D) \underbrace{\sum_{a=1}^{A} \mathrm{e}^{\mathrm{i} \kappa d_{a} \cdot s} q_{a}}_{F(s)} \mathrm{d} s .
$$

The choice of $L$ in eqn. (20), which is called the expansion length, has a significant influence on the accuracy and the performance of the multipole algorithm. Proper choice helps to circumvent divergence of the series and will be discussed later in this section. The sum on the right hand of (21) is called the far-field signature $F(s)$. It is local to the cluster with the sources $q_{a}$, since only the vector $d_{a}$ appears. In contrast to this, the translation operator $M_{L}$ only depends on the vector $D$ between 
two clusters' centers. Thus, if a regular cluster grid is used, the translation operators can be reused. Translating the far-field signature to another cluster using a translation operator forms the so called near-field signature. The solution is finally recovered by an exponential function of $d_{b}$ and an integration over the unit sphere.

Since the multipole expansion is only valid for well separated load and field points, one has to split up the clusters into a near-field and far-field. All clusters which fulfill the condition

$$
|D|<c_{d} \frac{d}{2}
$$

form the near-field. Here, $d$ denotes the cluster diameter and $c_{d}$ is a constant. The arising near-field is represented by a sparse matrix. It has to be evaluated by classical BEM. All other clusters are in the far-field and form the so called interaction list.

To obtain an optimal efficiency, a hierarchical multilevel cluster tree is used. It is set up by consecutive bisectioning such that a mother cluster is divided into two son clusters on the next level. The procedure starts with the root cluster, which is the smallest parallelepiped containing all elements of the model. The division is stopped if a specified number of elements per cluster is reached. These final clusters, which do not have any sons, are called leaf clusters. The interaction list of every cluster is formed by those clusters, which are in the near-field of the mother cluster but not in its own near-field.

Obviously, the far-field signature has to be translated to the interaction lists on different levels. Since the cluster diameters are different on every level, the expansion length $L$ has to be adapted to every level, too. Typically the well-established semi-empirical rule

$$
L\left(\kappa d_{\ell}\right)=\kappa d_{\ell}+c_{\mathrm{e}} \log \left(\kappa d_{\ell}+\pi\right)
$$

is used to estimate the number of series terms on level $\ell$ of the cluster tree [6]. The parameter $c_{e}$ has to be chosen by the user and determines the desired accuracy. In order to maintain the accuracy of the multipole expansion when the cluster diameter increases on the next level, an interpolation and filtering strategy has to be applied. It is advantageous to use a fast Fourier transform for this purpose. This is because new far-field directions have to be added, which is only possible for the original form of the multipole expansion $[7,8]$. The resulting fast multipole method (FMM) has a quasi linear complexity of order $\mathcal{O}\left(N \log ^{2} N\right)$ as outlined in [8].

The evaluation of the matrix-vector product is summarized with the following steps:

1. Compute the near-field part by a sparse matrix-vector multiplication.

2. Evaluate the far-field signature $F(s)$ for every leaf cluster.

3. Translate the far-field signature to all interaction clusters by means of the translation operators (Eq. 20) and sum it up as the near-field signature $N(s)$ there.

4. Shift the far-field signature to the mother cluster and repeat step 3 until the interaction list is empty. 
5. Go the opposite direction and shift the near-field signature $N(s)$ to the son clusters until the leaf clusters are reached.

6. Recover the solution by integration over the unit sphere.

\section{Coupled problem}

For the coupling of the FE formulation with the BE formulation, matching grids are considered. The first transmission condition eqn. (5) links the acoustic pressure $p$ with the tractions $t_{\mathrm{f}}$ of the structure and reads in matrix notation

$$
\boldsymbol{f}_{\mathrm{f}}=-\boldsymbol{C}_{\mathrm{FE}} \boldsymbol{p}
$$

where $\boldsymbol{C}_{\mathrm{FE}}$ is assembled of the element matrices

$$
\boldsymbol{C}_{\mathrm{FE}}^{k}=-\int_{\tau_{k}} \boldsymbol{N}_{u}^{\mathrm{T}} \boldsymbol{n}^{k} \boldsymbol{N}_{p} \mathrm{~d} s_{x} .
$$

Here, the matrices with the shape functions of the structure and the fluid are denoted by $N_{u}$ and $N_{p}$, respectively. A lumped force loading is applied, which neglects moments.

Due to the second transmission condition eqn. (6), the acoustic flux $q$ on each boundary element $\tau_{m}$ is computed from the structural displacements of the adjacent nodes $k$ by

$$
\boldsymbol{q}=\boldsymbol{T}_{q} \boldsymbol{u}
$$

where each row corresponds to

$$
q_{m}=\frac{1}{3} \varrho_{\mathrm{f}} \omega^{2} \sum_{k \in m} \boldsymbol{u}_{k} \cdot \boldsymbol{n}^{m},
$$

with the fluid density $\varrho_{\text {f }}$. Using the FE system eqn. (8), the definition of $\boldsymbol{C}_{\mathrm{FE}}$ by eqn. (24) and the BE representation eqn. (17) yields the coupled system of equations for the scattering problem

$$
\underbrace{\left(\begin{array}{cc}
\boldsymbol{K}_{\mathrm{FE}} & \boldsymbol{C}_{\mathrm{FE}} \\
\boldsymbol{C}_{\mathrm{BE}}^{q} \boldsymbol{T}_{q} & \boldsymbol{K}_{\mathrm{BE}}
\end{array}\right)}_{\boldsymbol{K}^{q}}\left(\begin{array}{l}
\boldsymbol{u} \\
\boldsymbol{p}
\end{array}\right)=\left(\begin{array}{c}
0 \\
\boldsymbol{b}_{\mathrm{BE}}
\end{array}\right) .
$$

Equation (28) is rewritten using the Schur complement as

$$
\left(\boldsymbol{K}_{\mathrm{BE}}-\boldsymbol{C}_{\mathrm{BE}}^{q} \boldsymbol{T}_{q} \boldsymbol{K}_{\mathrm{FE}}^{-1} \boldsymbol{C}_{\mathrm{FE}}\right) \boldsymbol{p}=\boldsymbol{b}_{\mathrm{BE}} .
$$

The structural displacements are then computed in a postprocessing step. It is worth mentioning, that the excitation of the structure results from the incident field which only indirectly acts on the structure through the acoustic fluid. The coupled 
system (29) is solved using a preconditioned GMRES [9]. The matrix-vector products with $\boldsymbol{K}_{\mathrm{BE}}$ and $\boldsymbol{C}_{\mathrm{BE}}$ are efficiently evaluated using the fast multipole method. Please note, that only the computation of the far-field signatures is different for the two matrices, but all other multipole steps are done simultaneously. In every iteration step, the effect of $\boldsymbol{K}_{\mathrm{FE}}^{-1}$ on a vector has to be evaluated. In this work, a $\mathrm{LDL}^{\mathrm{T}}$ factorization is applied for this purpose. This way, the factorization has to be computed only once and is then reused in every iteration step. To accelerate the convergence of the GMRES solver, the one-way coupled system is applied, where $\boldsymbol{C}_{\mathrm{FE}}$ is set to zero. In this work, an ILU factorization of the near-field of $\boldsymbol{K}_{\mathrm{BE}}$ is applied for preconditioning.

\section{Numerical example}

As test example a spherical shell structure which is hit by an incident plane wave is considered. For this type of problem an analytical solution is available [10]. The elastic structure is modeled with the commercial FE package ANSYS using SHELL63 elements (12 $\triangle$ and $3986 \square$ ) with six degrees of freedom at each node. The mean element size is $0.28 \mathrm{~m}$ and the model consists of 3994 nodes. The sphere has a radius $r=5.00 \mathrm{~m}$ and a shell thickness $t=0.05 \mathrm{~m}$. Steel is assumed as linear elastic material (Young's modulus $E=207 \mathrm{GPa}$, Poisson's ratio $\nu=0.3$, density $\varrho_{\mathrm{s}}=7669 \mathrm{~kg} / \mathrm{m}^{3}$ ). The material data of water (density $\varrho_{\mathrm{f}}=1000 \mathrm{~kg} / \mathrm{m}^{3}$, speed of sound $c=1387 \mathrm{~m} / \mathrm{s}$ ) are applied for the simulation of the exterior acoustic fluid. In the following, the point on the sphere which is first hit by the incident plane wave is denoted by $\mathrm{A}$, whereas $\mathrm{B}$ is located directly on the opposite side.

First, the pressure and displacement amplitudes at node $\mathrm{A}$ are investigated within the frequency range $f \in[5 \mathrm{~Hz}, 100 \mathrm{~Hz}]$. Figure 4 compares the numerical results of the FMBE-FE approach with the analytical ones for both an elastic sphere and a rigid sphere. In case of a rigid sphere, $\boldsymbol{C}_{\mathrm{FE}}$ in eqn. (29) is simply set to zero. Obviously, the agreement between the numerical and the analytical results is quite good. Additionally, one can clearly see that the elasticity of the sphere has a strong influence on the pressure results, justifying the use of a fully coupled solution scheme. Only at very low frequencies $f<10 \mathrm{~Hz}$, the solutions for the elastic sphere and rigid sphere tend to the same value. In this range, there are hardly any deformations and only rigid body motions occur.

In a second scenario, the directivity pattern along a semicircle between $\mathrm{A}$ and $\mathrm{B}$ on the surface of the sphere is investigated. An angle $\beta=0$ corresponds to point $\mathrm{B}$ whereas $\beta=180^{\circ}$ characterizes point A. Again, the numerical results are compared to the analytical ones for both the elastic and the rigid case. The simulations are performed at $75 \mathrm{~Hz}$. The results are visualized in Fig. 5. In case of $\beta=0^{\circ}$, the ratio $\mathrm{p}^{\mathrm{s}} / \mathrm{p}^{\text {inc }}$ is the highest for both the elastic and rigid case. The elastic sphere additionally has two sidelobes which can not be observed in the rigid case. Generally, the values are higher in the elastic case than in the rigid case. Once again, the results of the FE-BE approach are almost identical with the analytical solution. 

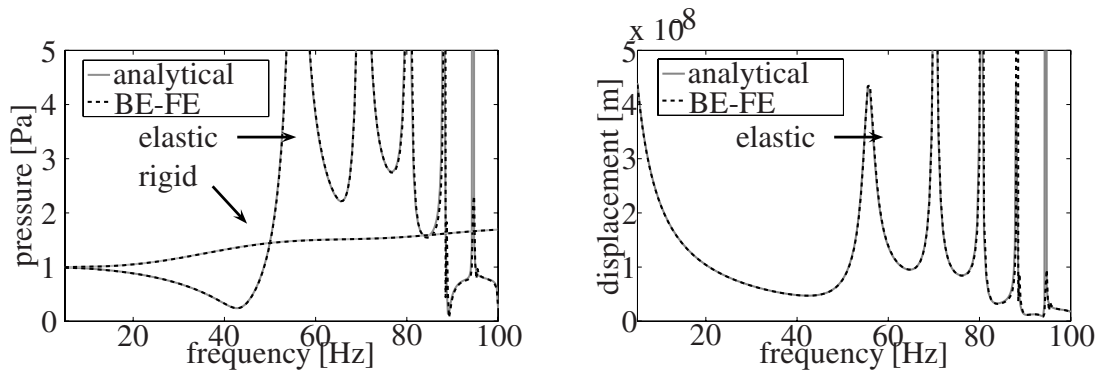

Figure 4: Sphere: pressure and displacement amplitudes at point A for a frequency sweep between 5 and $100 \mathrm{~Hz}$.

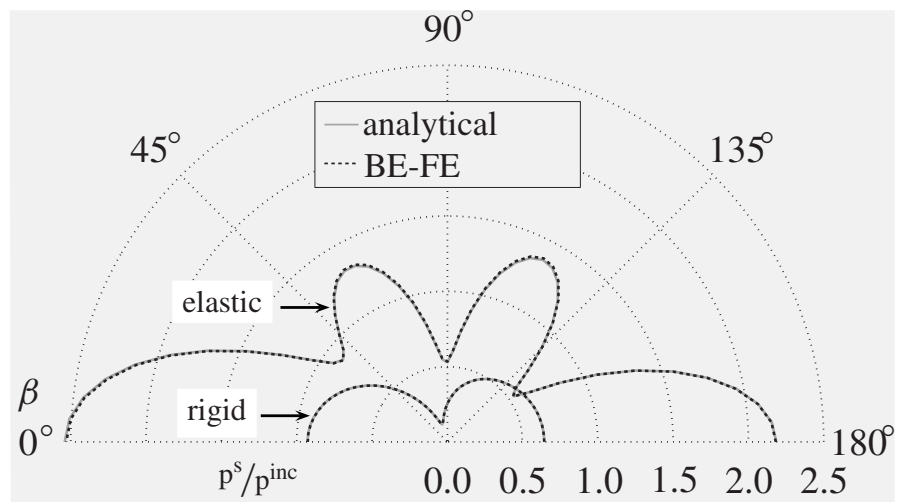

Figure 5: Directivity pattern of the spherical shell structure for $f=75 \mathrm{~Hz}$. The dimensionless pressure $\mathrm{p}^{\mathrm{s}} / \mathrm{p}^{\mathrm{inc}}$ is plotted for all points on a semicircle between points $\mathrm{A}$ and $\mathrm{B}$.

\subsection{Efficiency}

Concerning the simulation time, most of the time is spent with setting up the BE near-field matrices and with solving the obtained linear system. Proper preconditioning of the GMRES has a strong influence on the convergence rate. In this paper, a ILU preconditioner on $\boldsymbol{K}_{\mathrm{BE}}$ is applied to accelerate the GMRES. The required number of iterations steps for a tolerance of $10^{-6}$ is visualized in Fig. 6 (left). It is observable, that the number of iteration steps is lower in case of the rigid sphere, where it is almost independent of the frequency. This is not astonishing, since the structural part of the Schur complement is neglected for preconditioning. With increasing frequency, the influence of the elasticity of the structure increases. For a lower number of iterations, the second part of the Schur complement would need to be considered as well. 


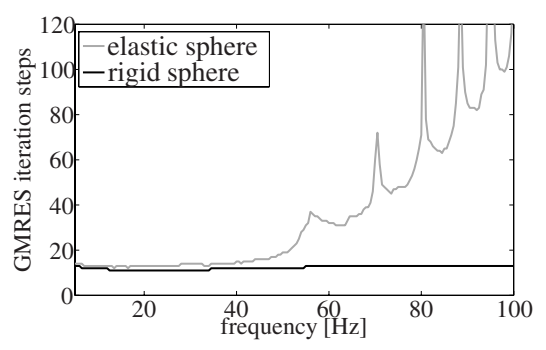

\begin{tabular}{|l|r|}
\hline & memory [MB] \\
\hline $\boldsymbol{K}_{\mathrm{BE}}$ (near-field) & 31.4 \\
\hline $\boldsymbol{C}_{\mathrm{BE}}$ (near-field) & 49.2 \\
\hline $\boldsymbol{K}_{\mathrm{FE}}$ & 7.6 \\
\hline $\boldsymbol{K}_{\mathrm{FE}}$ LDL $^{\mathrm{T}}$ & 57.8 \\
\hline $\boldsymbol{C}_{\mathrm{FE}}$ & 1.0 \\
\hline $\boldsymbol{T}_{u q}$ & 0.6 \\
\hline
\end{tabular}

Figure 6: Sphere: number of GMRES iteration steps with ILU preconditioning (left) and memory consumption for the submatrices (right).

The memory consumption of all matrices is summarized in the table on the right hand side of fig. 6 . The sparse BE near-field matrices are more expensive than the sparsely populated FE system matrix. The memory consumption of $\boldsymbol{C}_{\mathrm{FE}}$ and $\boldsymbol{T}_{u q}$ is negligible. The significant part of the memory consumption is the $\mathrm{LDL}^{\mathrm{T}}$ factorization of the dynamic stiffness matrix $\boldsymbol{K}_{\mathrm{FE}}$, which is symmetric and real valued in absence of structural damping. Theoretically, one could apply an iterative solver for $\boldsymbol{K}_{\mathrm{FE}}^{-1}$ to avoid the factorization. But as a consequence the efficiency would decrease because of the resulting nested solution scheme. Additionally, proper preconditioning of $\boldsymbol{K}_{\mathrm{FE}}$ which typically results from shell elements is a non-trivial task.

\section{Conclusion}

In this paper scattering problems with fluid-structure interaction are investigated. For this purpose, a conforming coupling formulation between the boundary element method and finite element method is presented. For the finite element part, an interface to a commercial code is set up. The boundary element part is accelerated by the fast multipole method. Thus, even large scale problems can efficiently be computed. Numerical results for a spherical shell structure with an incident plane wave are compared with an analytical solution and show a good accuracy of the applied FE-BE approach. Additionally, the influence of the elasticity of the structure turned out to have a strong effect on the overall results. This necessitates the use of a strong coupling scheme. The overall computation time is influenced by proper preconditioning which should be further improved for an optimal performance.

\section{Acknowledgements}

This research was financially supported by the German Research Foundation (DFG) under the transfer project SFB404/T3. The authors acknowledge valuable contributions of Germanischer Lloyd AG, Hamburg within this transfer project. 


\section{References}

[1] Gaul, L., Kögl, M. \& Wagner, M., Boundary Element Methods for Engineers and Scientists. An Introductory Course with Advanced Topics. Springer Berlin, 2003.

[2] Wu, T.W., (ed.) Boundary Element Acoustics. Fundamentals and Computer Codes. WIT Press Southampton, 2000.

[3] Ansys, Guide for Interfacing with Ansys: Release 10.0, 2005.

[4] Burton, A.J. \& Miller, G.F., The application of integral equation methods for the numerical solution of boundary value problems. Proc Roy Soc London A, 232, pp. 201-210, 1971.

[5] Rokhlin, V., Diagonal forms of translation operators for the Helmholtz equation. Appl Comput Harmon A, 1, pp. 82-93, 1993.

[6] Coifman, R., Rokhlin, V. \& Wandzura, S., The fast multipole method for the wave equation: A pedestrian description. IEEE Antenn Progag $M, \mathbf{3 5}$, pp. 7-12, 1993.

[7] Gyure, M.F. \& Stalzer, M.A., A prescription for the multilevel Helmholtz FMM. IEEE Comput Sci Eng, 5, pp. 39-47, 1998.

[8] Fischer, M., The Fast Multipole Boundary Element Method and its Application to Structure-Acoustic Field Interaction. Ph.D. thesis, University of Stuttgart, 2004.

[9] Brunner, D., Junge, M. \& Gaul, L., A comparison of FE-BE coupling schemes for large scale problems with fluid-structure interaction. Int J Numer Meth Eng, in press, DOI: 10.1002/nme.2412, 2008.

[10] Junger, M.C. \& Feit, D., Sound, Structures, and Their Interaction: Basic Concepts. The MIT Press, 2nd edition, 1986. 